\title{
Resistance of Blueberry Cultivars to Botryosphaeria Stem Blight and Phomopsis Twig Blight
}

\author{
James J. Polashock ${ }^{1}$
}

USDA-ARS Fruit Lab, P.E. Marucci Center for Blueberry and Cranberry

Research, 125A Lake Oswego Road, Chatsworth, NJ 08019

\section{Matthew Kramer \\ USDA-ARS Biometrical Consulting Service, Henry A. Wallace Agricultural Research Center, Beltsville, MD 20705}

Additional index words. stem disease, Botryosphaeria dothidea, Phomopsis vaccinii, Vaccinium spp.

\begin{abstract}
Stem diseases of blueberry (Vaccinium spp.) can cause significant crop loss as well as loss of entire bushes. Stem diseases are also more difficult to control with fungicides than foliar or fruit diseases. A screening program was initiated to test blueberry cultivars for resistance to two pathogenic fungi: botryosphaeria stem blight and phomopsis twig blight. An attached stem assay was developed to compare the host response with both fungi. The relative resistance of $\mathbf{5 0}$ blueberry cultivars was assessed using stem lesion lengths, analyzed on a log scale, taken at 4 weeks postinoculation. For Botryosphaeria stem blight, mean lesion length ranged from about $10 \mathrm{~mm}$ in resistant cultivars to about $140 \mathbf{~ m m}$ in susceptible cultivars. The half-high cultivars Northsky, Northblue, and Chippewa, and the lowbush cultivar Putte were among the most resistant. Phomopsis twig blight lesions ranged in mean length from about 18 to $98 \mathrm{~mm}$. Similar to results for Botryosphaeria stem blight, resistance was limited to half-high ('Northsky' and 'Chippewa') and lowbush ('Blomidon', 'Chignecto', and 'Cumberland') cultivars. Individual cultivars resistant to one pathogen were not necessarily resistant to the other; although, overall, the resistances were correlated. Approximate 95\% confidence intervals were established for all cultivars to predict mean performance across years. The cultivars tested varied in resistance, but the largest single factor affecting lesion length was the fungal isolate used for inoculations. These data enable us to identify cultivars resistant to both diseases that can be used for planting in problem areas, as well as selection of parental material for breeding cultivars with improved resistance.
\end{abstract}

Several blueberry species are affected by the stem diseases botryosphaeria stem blight [Botryosphaeria dothidea (Moug.:Fr.) Ces. $\&$ De Not] and phomopsis twig blight (Phomopsis vaccinii Shear). Symptoms of Botryosphaeria stem blight include reddening and drying of leaves on affected shoots. The entire shoot eventually dies back, and the infection can progress into the crown, killing entire bushes (Milholland, 1995a; 1972; Witcher and Clayton, 1963). Primary sites of infection are pruning wounds and sites of winter injury. Previous work has described methods of inoculation to determine susceptibility of whole plants to $B$. dothidea (Cline et al., 1993; Creswell and Milholland, 1987) and in vitro shoots (Smith, 2004). Field resistance has been reported for the cultivars Murphy, Cape Fear, and O'Neal; however, there is little information on resistance to this disease for many of the cultivars currently available.

\footnotetext{
Received for publication 27 Apr. 2006. Accepted for publication 22 June 2006. We thank Kristia Adams and Allan Stretch for technical assistance. ${ }^{1}$ To whom reprint requests should be addressed; e-mail jpolashock@ars.usda.gov
}

Wilting of leaves on affected shoots and browning of flower buds characterize phomopsis twig blight (Milholland, 1982; Wilcox, 1939). Under certain conditions, $P$. vaccinii can also cause fruit rot and canker (Milholland, 1995b; Ramsdell, 1995). A small number of highbush cultivars have been screened for resistance to the canker form of this disease (Baker et al., 1995), but like Botryosphaeria stem blight, resistance data for many blueberry cultivars are not available. The objectives of this project are 1) to determine the relative resistance of blueberry cultivars to botryosphaeria stem blight and phomopsis twig blight infection, and 2) to establish the impact of pathogen strain variability on cultivar response to infection.

\section{Materials and Methods}

\section{Plant material}

Fifty cultivars were used in this study from the following blueberry types: highbush (Vaccinium corymbosum L.), southern highbush (southern-adapted $V$. corymbosum, usually introgressed with $V$. darrowi Camp), lowbush ( $V$. angustifolium Ait.), half-high ( $V$. corymbosum $\times V$. angustifolium hybrids), and rabbiteye ( $V$. ashei Reade). The cultivars used in this study, types, and abbreviations used in the figures are shown in Table 1. All blueberry plants were 3 to 5 years old and were maintained in cold frames in 3-L pots. Plants were pruned to the crown in early spring. After regrowth, plants with at least two to three uniform shoots suitable for inoculation were selected in late August and moved to a greenhouse. All plants were partially hardened at the time of inoculation. Partially hardened succulent stems are reported to reflect cultivar susceptibility more accurately than either very young or older stems (Creswell and Milholland, 1987). In 2003, inoculations were made on 11 Sept. (botryosphaeria stem blight) and 16 Sept. (phomopsis twig blight). The experiment was repeated in 2004 with inoculation dates of 16 Sept. and 22 Sept. for stem blight and twig blight respectively. In 2005, inoculations for each fungus were split between two days (19, 20 Sept. for B. dothidea and 21, 22 Sept. for $P$. vaccinii).

\section{Fungal isolates}

All fungal isolates were from infected blueberry tissue, and isolations were made 1 month before inoculation, as recommended by Cline et al. (1993). For botryosphaeria stem blight, three isolates of $B$. dothidea were collected from research fields at the P.E. Marucci Center for Blueberry and Cranberry Research, in Chatsworth, N.J. (2003), and from a commercial farm in Atlantic County, N.J. (2004). For phomopsis twig blight, two isolates of $P$. vaccinii were collected from the field in Chatsworth, N.J., in 2003 and 2004. In 2005, a much larger number of fungal isolates was used (16 of each fungal species), collected from various locations in Atlantic and Burlington counties, N.J., and from various highbush blueberry cultivars. All fungal isolates were maintained on potato dextrose agar (PDA; $0.4 \%$ potato starch, $2.0 \%$ dextrose, $1.5 \%$ agar).

\section{Inoculation}

The inoculation method used was similar to that developed by Creswell and Milholland (1987) for B. dothidea. We reasoned that because $P$. vaccinii is also a wound pathogen, the same inoculation technique could be used for both fungi. Fungal isolates were grown in a $22{ }^{\circ} \mathrm{C}$ incubator on PDA plates. Agar plugs were punched from the plates with a no. 3 cork borer. Stems to be inoculated were selected to be of similar diameter within a cultivar and inoculations were limited to the tip of the second flush. Shoot tips were removed with pruning shears and a pathogencontaining agar plug was placed myceliumside down on the fresh-cut surface. Diameter of all treated stems was measured using digital calipers at a point just below the inoculated surface. Uninfected controls were similarly treated except that the agar plugs were taken from sterile PDA plates. Inoculation sites were immediately covered with parafilm (Pechiney Plastic Packaging, Chicago) to prevent desiccation. The film was removed after $3 \mathrm{~d}$. Lesion length, as 
Table 1. Blueberry cultivars used in screening for resistance to botryosphaeria stem blight and phomopsis twig blight, blueberry types, and cultivar abbreviations.

\begin{tabular}{|c|c|c|c|c|c|}
\hline Cultivar & Type $^{z}$ & Abbr. ${ }^{y}$ & Cultivar & Type & Abbr. \\
\hline Ascorba & HB & ASC & Gretha & $\mathrm{HB}$ & GRT \\
\hline Berkeley & HB & BER & Jersey & HB & JER \\
\hline Blomidon & LB & BLO & Lateblue & $\mathrm{HB}$ & LAB \\
\hline Bluecrop & $\mathrm{HB}$ & $\mathrm{BC}$ & Millennium & SHB & MIL \\
\hline Bluejay & HB & BJA & Murphy & HB & MUR \\
\hline Blueray & HB & BRA & Nelson & HB & NEL \\
\hline Cape Fear & SHB & CAF & Northsky & $\mathrm{HH}$ & NOS \\
\hline Cara's Choice & HB & $\mathrm{CAC}$ & O'Neal & $\mathrm{HB}$ & ONE \\
\hline Chignecto & LB & CHG & Ozarkblue & SHB & OZB \\
\hline Chippewa & $\mathrm{HH}$ & CHP & Polaris & $\mathrm{HH}$ & POL \\
\hline Collins & $\mathrm{HB}$ & $\mathrm{COL}$ & Powderblue & $\mathrm{RE}$ & PWB \\
\hline Coville & HB & $\mathrm{COV}$ & Putte & LB & PUT \\
\hline Cumberland & LB & CUM & Reveille & SHB & REV \\
\hline Fundy & LB & FUN & Sunrise & HB & SNR \\
\hline Gila "1876" & HB & GIL & Weymouth & HB & WEY \\
\hline Goldtraube & HB & GOT & Zuckertraube & LB & ZUK \\
\hline
\end{tabular}

${ }^{\text {}}$ Blueberry type as described in the text: HB, highbush; LB, lowbush; SHB, southern highbush; HH, half-high; RE, rabbiteye.

${ }^{y}$ Cultivar abbreviations used in Figs. 1 and 2.

determined by the distance between the inoculation point and the edge of the necrotic zone, was recorded at 2 weeks and 4 weeks after inoculation. Plants were maintained in a greenhouse at ambient temperatures after inoculation and until measurements were completed. For botryosphaeria stem blight, inoculation with each of three fungal isolates was replicated three times per cultivar $(2003$ and 2004) for a total of nine stems per cultivar each year. For phomopsis twig blight inoculations, plant material was limited, and each of two fungal isolates was replicated two times per cultivar (2003 and 2004) for a total of four stems per cultivar each year.

Preliminary analysis of 2003 and 2004 data suggested a high level of variability in virulence of fungal isolates. Therefore, in 2005 , a wide array of isolates was used to assess better the isolate variability and to test for a possible isolate $\times$ cultivar interaction. Each cultivar was inoculated in an incomplete block design, with nine different fungal isolates selected at random from the collection of 16 isolates. In 2005, to conserve stems for pathogen inoculations, uninfected controls were eliminated because in previous years (2003 and 2004) the necrotic portion of the stem in the controls (typically only $1-$ $3 \mathrm{~mm}$ long) was limited to that incited by the pruning wound.

To verify that models for the analysis of the incomplete block design with all cultivars were reasonable, we ran additional replicates for two cultivars to estimate within-isolate variances independently. The cultivars Bluejay and Elliott for $B$. dothidea, and Duke and Polaris for $P$. vaccinii were selected because they differed in susceptibility during the first 2 years of the study. These plants were inoculated 12 times each with two randomly selected isolates from the collection.

\section{Statistical analysis}

The dependent variable for both fungal pathogens was the length of the lesion (in millimeters). Plots of mean lesion length versus SD (grouped by cultivar and isolate) suggested that the SD was a function of the mean, thus requiring transformation to meet the assumption for valid $\mathrm{F}$ and $t$ tests that variances are homogeneous across the groups. We used the transformation $\mathrm{z}=\log$ $(y+1)$, where $y$ is lesion size, which removed this dependence. Although statistical comparisons and confidence intervals were performed on transformed data, the results were back-transformed for figures and tables for ease of interpretation.

We analyzed the data in a mixed models framework using SAS PROC MIXED (SAS version 9.1; SAS Institute, Cary, N.C.), maximizing the restricted likelihood, which produces residual maximum likelihood (REML) parameter estimates. We examined models with a variety of fixed and random effects to determine whether stem diameter was a useful covariate and whether there was an interaction between isolate and cultivar. $A$ posteriori $P$ value adjustments for multiple comparisons were made using the TukeyKramer method (available in the SAS PROC MIXED software). Our final statistical models for both pathogens included the same fixed and random effects.

We used a three-category system to create easily understood groups of cultivars based on their resistance to each pathogen. Cultivars were grouped by whether their mean (least squares mean) lesion length was significantly less than, greater than, or not different from the average of all cultivars. For these means comparisons, we chose the cultivar with a mean lesion length that was closest to the mean lesion length of all cultivars and then used a $t$-test to determine whether each of the other cultivars differed from it. An $\alpha$ of 0.05 produced three groups, with only a few cultivars in the two outer groups (i.e., cultivars that differed from average). A more liberal $\alpha=0.10$ adds a few cultivars to the outer groups and may produce a more useful three-category grouping for selection of resistant cultivars.

Approximate $95 \%$ confidence intervals around the mean were constructed by adding or subtracting twice the SEM. When backtransformed to the original scale, as shown in the figures and tables, this creates asymmetrical confidence intervals.

To estimate the importance of the various effects in the model, we used standard variance decomposition techniques (Searle et al., 1992) and the $R_{M L}^{2}$ for mixed models (Kramer, 2005) as a measure of model fit and to determine the relative contribution of the fixed and random effects. We report $P$ values from Wald statistics for the random effects, from the SAS PROC MIXED output.

The Pearson correlation coefficient was used to compare our results with those published from other studies. Methodologies used in the other studies sometimes differed from ours and analysis was necessarily limited to cultivars in common. We also used the Pearson correlation coefficient for betweenyear comparisons of our results, as a benchmark for expected consistency.

\section{Results}

\section{Botryosphaeria stem blight}

Data acquired 2 weeks postinoculation did not allow for clear separation of cultivar susceptibility, presumably because the pathogen had insufficient time to colonize the stems. These experiments were ended at 
4 weeks postinoculation because the lesions in more susceptible plants were expected to reach the crown by week 6 . Data acquired at to determine relative resistance to this pathogen. Botryosphaeria stem blight lesions on individual plants varied from 0 (i.e., no lesion) to $404 \mathrm{~mm}$ in length. Back-transformed least squares means from the model (across all 3 years) were between $12 \mathrm{~mm}$ and about $55 \mathrm{~mm}$ (Fig. 1). Within-cultivar variation was high, primarily as a result of isolate differences, but also because of year-to-year variation and stem-to-stem differences. The approximate $95 \%$ confidence intervals on the means (Fig. 1) indicate the expected performance of the cultivars under varying conditions, including isolate differences.

The blueberry types (i.e., highbush, southern highbush, etc., as described earlier and listed in Tables 1 and 2) generally span the range; however, the half-high cultivars cluster was significantly more resistant (Table 2). 'Friendship' was the only half-high cultivar that showed moderate resistance $(33 \mathrm{~mm})$, but it should be noted that this cultivar is a wild selection from Wisconsin of unknown parentage. Among highbush cultivars, 'Weymouth' $(20 \mathrm{~mm})$ was the most resistant whereas 'Duke' (55 mm) and 'Bluecrop' (55 mm) were among the most susceptible. In 2003 and 2004, the highbush cultivar Reka was among the most resistant (data not shown), but limited plant material did not allow for testing in 2005, so this cultivar was not included in Fig. 1.

\section{Phomopsis twig blight}

Similar to stem blight, data acquired 2 weeks postinoculation did not allow for clear 4 weeks postinoculation were therefore used

separation of cultivar response to infection. This fungus grew much more slowly than $B$. dothidea, and measurements were extended to week 6 . A preliminary analysis of the data indicated that relative differences in lesion length between weeks 4 and 6 were similar (data not shown), and data taken at 4 weeks postinoculation were therefore used for the statistical analyses.

Phomopsis twig blight lesions (at week 4 postinoculation) on individual plants ranged from 0 to $222 \mathrm{~mm}$. The back-transformed least squares means ranged from 17 to $58 \mathrm{~mm}$ (Fig. 2). The cultivars that were the most resistant were the lowbush 'Chignecto' (17 $\mathrm{mm})$ and the half-high 'Chippewa' (17 mm). The southern highbush 'Emerald' (58 mm) and the rabbiteye 'Powderblue' $(48 \mathrm{~mm})$ were the most susceptible varieties. Types of cultivars separated similarly to those seen for botryosphaeria stem blight, with halfhigh cultivars showing significantly more resistance on average than other types (Table $2)$. There was a significant positive correlation between resistances to the two pathogens $(r=0.44, P=0.001)$, based on the least squares means of the cultivars.

\section{Variance decomposition}

The model estimates of the residual variances were very close to our independent estimates based on the 12 replicates per isolate-cultivar combinations. This indicates that the model adequately captured the variability resulting from the experimental factors.

The $R_{\mathrm{mL}}^{2}$ for our mixed model for botryosphaeria stem blight was 0.55 , which was decomposed into a contribution of 0.11 by the fixed part and 0.44 by the random part. For

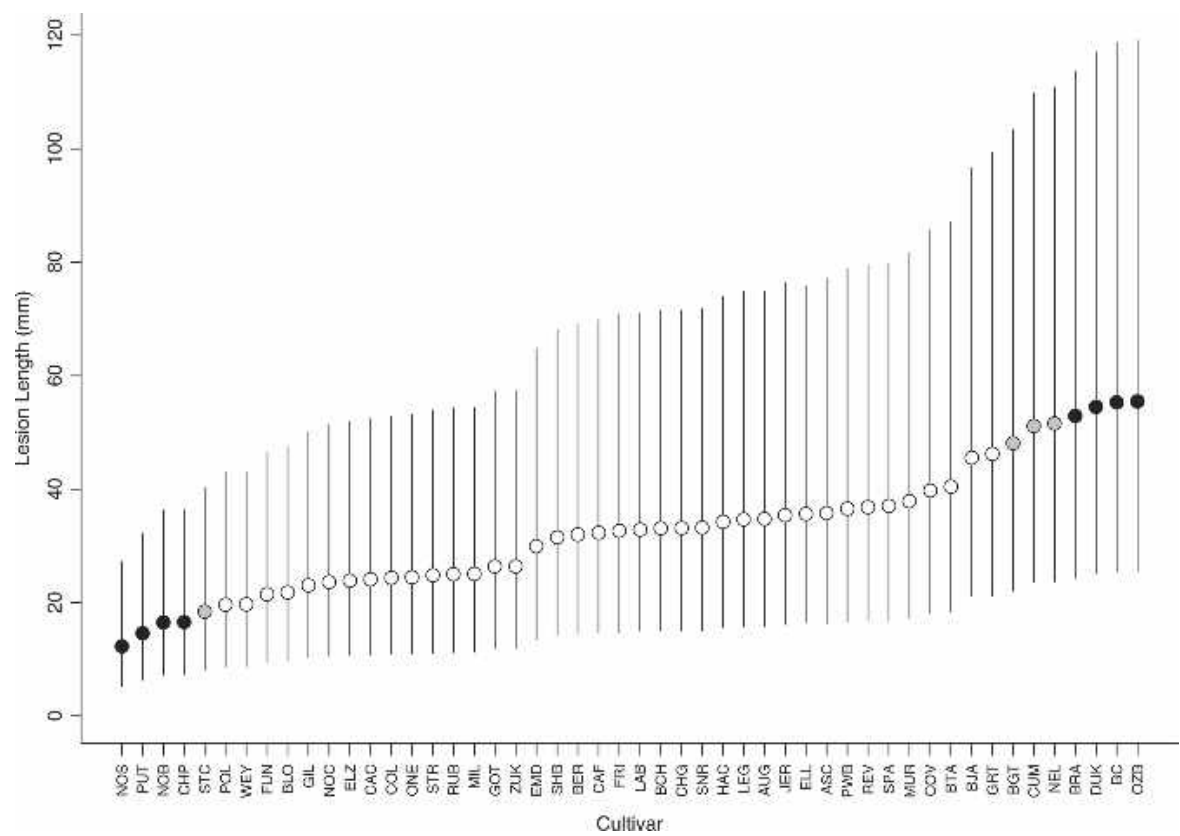

Fig. 1. Response of blueberry cultivars to Botryosphaeria dothidea infection 4 weeks postinoculation. Cultivar abbreviations are as listed in Table 1. Shown are mean lesion lengths (circles) and approximate $95 \%$ confidence intervals (lines) on the means for each cultivar tested. Cultivars with black circles are different from the average (across all cultivars) at $P=0.05$. Cultivars with gray or black circles are different from the average at $P=0.1$. phomopsis, the $\mathrm{R}_{\mathrm{ML}}^{2}$ of 0.68 , decomposed into 0.12 (fixed) and 0.56 (random). Stem diameter was not a significant predictor of lesion length for either pathogen $(P>0.05)$ and was not included in the models. Our models contained two sets of fixed effects: blueberry type and cultivar nested in type. We found significant differences among types and among cultivars nested within types (all $P<0.05$ ).

We included the random effects of isolate, year, and cultivar $\times$ year interaction in our modeling. We did not find an isolate $\times$ cultivar interaction random effect for either pathogen $(P>>0.05$, Wald test). Thus, the responses of cultivars were similar across the isolates. However, the year effect was not the same on all cultivars (i.e., the apparent resistance of a cultivar might change from one year to the next - a cultivar $\times$ year interaction).

The cultivar $\times$ year effect was investigated by comparing results of the years analyzed separately (results not shown; the average correlation between years is given in the following section). We found that this interaction effect was spread throughout the cultivars and resulted in minor ordering changes throughout the 3 years, but had little effect on the size of the confidence intervals. In any case, the year and cultivar $\times$ year effects were much smaller than the isolate effect, which was the largest component of the random effects.

The isolate effect alone was responsible for about $56 \%$ of the explained variation in lesion length for botryosphaeria stem blight and about $80 \%$ for phomopsis twig blight. Table 3 gives a variance decomposition for the random effects for the two pathogens. The overall year effect was estimated to be nonsignificant for botryosphaeria stem blight and zero for the phomopsis twig blight data set, but the year $\times$ cultivar interaction was significant, so the year term was retained.

\section{Comparison with other studies}

For both pathogens, all between-year correlations of cultivar least square means (based on 50 cultivars $)$ were significant $(P<0.01)$, with averages of $r=0.46$ for both years. Comparisons with other studies were necessarily based on far fewer cultivars. We used the overall (throughout the 3 years) least squares means for these comparisons. For botryosphaeria stem blight, our results agreed closely with those of Creswell and Milholland (1987), based on seven cultivars $(r=0.87$, $P=0.025)$. There were eight cultivars available for a comparison with Smith (2004, 30-d results). Results from that study did not agree with ours $(r=0.07, P=0.88)$. For phomopsis twig blight, our results differ from those of Baker et al. (1995). With seven cultivars available for comparison (and using their mortality rate estimates, which should yield a correlation coefficient with a positive sign), we calculated $r=-0.11(P=0.82)$.

\section{Discussion}

Resistance to both of the pathogens, as determined by differences in mean lesion 
Table 2. Mean lesion length and approximate $95 \%$ confidence intervals for comparison of blueberry types for resistance to botryosphaeria stem blight and phomopsis twig blight.

\begin{tabular}{lccccc}
\hline & \multicolumn{2}{c}{ Botryosphaeria stem blight } & & \multicolumn{2}{c}{ Phomopsis twig blight } \\
\cline { 2 - 3 } \cline { 5 - 6 } Type $^{\mathrm{z}}$ & $19.2 \mathrm{a}$ & $95 \% \mathrm{CI}^{\mathrm{x}}$ & & Mean lesion length & $95 \% \mathrm{CI}$ \\
\hline Half-high & $27.1 \mathrm{~b}$ & $9.4,38.3$ & & $2.2 \mathrm{a}$ & $15.3,32.0$ \\
Lowbush & $34.2 \mathrm{bc}$ & $13.4,53.7$ & & $24.3 \mathrm{~b}$ & $16.8,35.0$ \\
Highbush & $34.8 \mathrm{c}$ & $17.3,66.7$ & & $31.8 \mathrm{c}$ & $22.4,45.0$ \\
Southern highbush & $36.5 \mathrm{c}$ & $16.5,68.8$ & & $33.1 \mathrm{~cd}$ & $23.0,47.5$ \\
Rabbiteye & 36.3 & & $48.4 \mathrm{~d}$ & $30.7,76.0$ \\
\hline
\end{tabular}

${ }^{2}$ Blueberry type as described in the text and noted for each cultivar in Table 1.

${ }^{y}$ Means followed by the same letter do not differ significantly. Estimates for Rabbiteye are based on only one cultivar Powderblue and may not be representative of the whole species ( $V$. ashei)

${ }^{\mathrm{x}}$ Approximate $95 \%$ confidence intervals (CI) on mean lesion length (in millimeters).

length, varied across cultivars. Mean lesion length for botryosphaeria stem blight varied $\approx 4$-fold across all cultivars, whereas phomopsis twig blight mean lesion length varied 3.5-fold. Response to infection was nearly a continuum, as might be expected considering the large number of cultivars that were screened. None of the cultivars were found to be immune to either disease, and resistance to one of the pathogens did not always indicate resistance to the other (e.g., 'Cumberland'). However, there was a significant correlation between resistances to the two pathogens ( $r=$ $0.44, P=0.001)$, suggesting some commonality in the mechanism of resistance (Table 2 ). The source of resistance in these cultivars is not obvious, because many are interspecific hybrids. However, one commonality in overall resistance is the presence of lowbush ( $V$. angustifolium) germplasm. Of the species tested for resistance to stem blight by Buckley (1990), $V$. angustifolium exhibited the best potential as a source of resistance. Some resistance was also shown for $V$. arboreum, whereas $V$. corymbosum lacked resistance

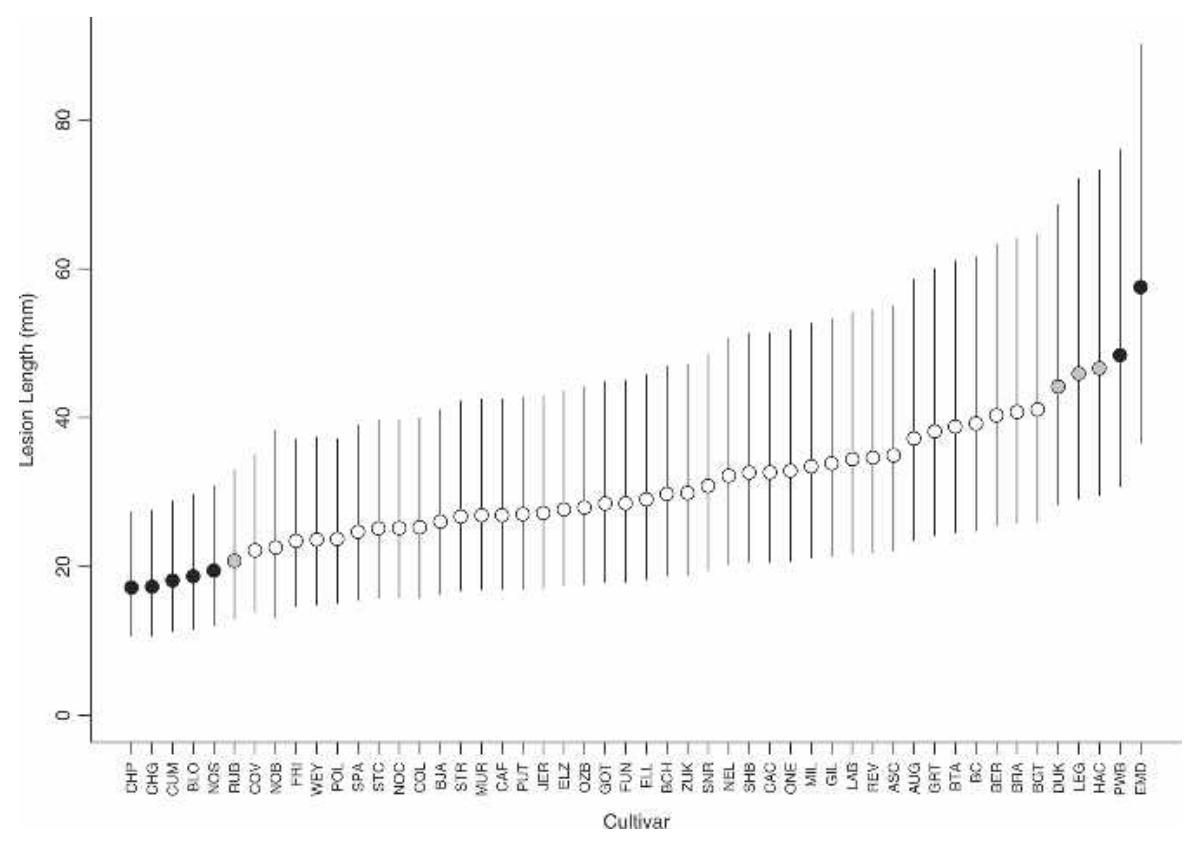

Fig. 2. Response of blueberry cultivars to Phomopsis vaccinii infection 4 weeks postinoculation. Cultivar abbreviations are as listed in Table 1 . Shown are mean lesion lengths (circles) and approximate $95 \%$ confidence intervals (lines) on the means for each cultivar tested. Cultivars with black circles are different from the average (across all cultivars) at $P=0.05$. Cultivars with gray or black circles are different from the average at $P=0.1$. cultivars near the mean. Our data agreed well with Creswell and Milholland (1987), on which the technique we used was based.

Baker et al. (1995) screened nine highbush cultivars for resistance to phomopsis canker and listed 'Elliott' and 'Bluetta' as the most resistant of the cultivars tested. Phomopsis vaccinii is the causal agent of both phomopsis twig blight and phomopsis canker, and the stem inoculations used by Baker et al. (1995) caused lesions, but no cankers. In our study, 'Elliott' was near the mean for response to $P$. vaccinii infection across all cultivars tested, and 'Bluetta' was among the most susceptible.

Inoculation method, isolate, age of the isolate, and assumptions in the various statistical approaches differ between these studies and ours, which could lead to contradictory rankings. One issue is the statistical treatment of isolate as a random effect or fixed effect. Had isolates been treated as random effects in previous studies, fewer significant differences in cultivar response would have been reported. Furthermore, in our study, ranking agreement among years was only moderate, which contributes to the uncertainty of determining "true" cultivar resistance. Most other studies reported only one year of data.

Only Creswell and Milholland (1987) reported an interaction effect between isolate and cultivar. Had they considered isolate as a random variable, this interaction effect might well have dropped to nonsignificance. An interaction of cultivar with isolate would make the results specific for the isolate present at the time. Screening under a significant cultivar $\times$ isolate interaction would entail testing large numbers of pathogen strains. Thus, if many cultivars need to be tested with a large number of isolates, the screening process would quickly become unmanageable.

We are encouraged by the significant correlations over years and between studies using the same methods, suggesting that this methodology is able, reliably, to identify resistant cultivars. We are less encouraged when different methods yield different rankings, because it then becomes questionable which methodology is best for determining practical resistance.

The use of $R^{2}$ and variance decomposition was useful to show how the independent variables contribute to the observed variation in lesion length. Thus, although cultivar differences were significant, they were small compared with the overall differences in virulence among the isolates. This is in agreement with conclusions of Cline et al. (1993) that there is a high degree of pathogen variability, but fairly constant relative levels of cultivar susceptibility. The year effect (including the year $\times$ cultivar interaction) was larger for stem blight, indicating that there is some uncertainty in ranking when averaging over years. This effect, which includes environmental differences among years and yearly pathogen virulence variation, would predicate the selection cultivars 
Table 3. Variance component estimates on a log-transformed scale for the random effects for botryosphaeria stem blight and phomopsis twig blight.

\begin{tabular}{|c|c|c|c|c|}
\hline \multirow[b]{2}{*}{ Random effect } & \multicolumn{2}{|c|}{ Botryosphaeria stem blight } & \multicolumn{2}{|c|}{ Phomopsis twig blight } \\
\hline & Estimate & $P$ value ${ }^{z}$ & Estimate & $P$ value \\
\hline Isolate & 0.6033 & 0.0012 & 0.5219 & 0.0009 \\
\hline Year & 0.1904 & 0.2601 & 0 & - \\
\hline Year $\times$ cultivar & 0.0684 & $<0.001$ & 0.0097 & 0.0325 \\
\hline Residual & 0.4452 & - & 0.1573 & - \\
\hline
\end{tabular}

${ }^{\mathrm{z}} P$ values are estimated using a Wald statistic.

that will be resistant in most years (i.e., those that have a high average resistance).

Although there is separation of cultivars based on their reaction to these pathogens, the single best predictor of an individual stem's response to inoculation with either pathogen is determined by which isolate was used. There is also a large proportion of unexplained variation (based on $\mathrm{R}_{\mathrm{ML}}^{2}$ ) $-45 \%$ for botryosphaeria and $32 \%$ for phomopsiswhich obscures cultivar differences. The large proportion of unexplained variation appears to be a common problem in screening experiments.

One explanation for why unexplained variance is high includes physical variation of the biological materials. For example, uniformity of plant material between cultivars is especially difficult when different blueberry types are screened (e.g., lowbush stems are much smaller in diameter than highbush stems). A more general problem is that of missing covariates or an incomplete knowledge of the system. For example, the pathogen's ability to create a large lesion may be based on physiological status of the stem at the time of inoculation. Plants that are genetically identical must adapt to both the general and local environments. Thus, stems and tissues from the same plant may have somewhat different histories and therefore a different response to infection. This may also be true of the isolates used; that is, a seemingly homogeneous mixture may actually be heterogeneous, resulting in inoculum variation, which would contribute to the error in cultivar resistance determination.

We found that the estimate of the stem-tostem variance from the heavily replicated within year-isolate-cultivar combinations was very similar to the residual variance in the mixed-models analysis, suggesting that at least the basic form of the statistical model was correct. To improve separation of cultivars, stem-to-stem variance must be reduced, and this should be one goal of future research.

The large differences in average lesion length among isolates suggests that another important research goal should be aimed at understanding why some isolates are so much more virulent than others. Additionally, when developing resistant cultivars, it will be important to use the most virulent isolates for testing. Unfortunately, as with many plant pathogens, the virulence of $B$. dothidea is reported to decline in culture (Cline et al., 1993), so using the same characterized pathogens across years is not yet practical.

\section{Literature Cited}

Baker, J.B., J.F. Hancock, and D.C. Ramsdell. 1995. Screening highbush blueberry cultivars for resistance to Phomopsis canker. Hort Science 30:586-588.
Buckley, B. 1990. Occurrence of stem blight resistance in blueberry PhD Diss., N.C. State Univ., Raleigh.

Cline, W.O., R.D. Milholland, S.D. Rooks, and J.R. Ballington. 1993. Techniques in breeding for resistance to blueberry stem blight caused by Botryosphaeria dothidea. Acta Hort. 346: 107-110.

Creswell, T.C. and R.D. Milholland. 1987. Responses of blueberry genotypes to infection by Botryosphaeria dothidea. Plant Dis. 71: 710-713.

Kramer, M. 2005. $R^{2}$ statistics for mixed models, p. 148-160. In: J. Boyer (ed.). Proc.17th Annu. Kansas State Univ. Conf. on Appl. Stat. in Agr., Kansas State Univ. Manhattan, Kans.

Milholland, R.D. 1982. Blueberry twig blight caused by Phomopsis vaccinii. Plant Dis. 66:1034-1036.

Milholland, R.D. 1995a. Botryosphaeria stem blight, p. 10-11. In: F.L. Caruso and D.C. Ramsdell (eds.). Compendium of blueberry and cranberry diseases. APS Press, St. Paul, Minn.

Milholland, R.D. 1995b. Phomopsis twig blight and fruit rot, p. 13-14. In: F.L. Caruso and D.C. Ramsdell (eds.). Compendium of blueberry and cranberry diseases. APS Press, St. Paul, Minn.

Milholland, R.D. 1972. Histopathology and pathogenicity of Botryosphaeria dothidea on blueberry stems. Phytopathology 62:654-660.

Ramsdell, D.C. 1995. Phomopsis canker. APS Press, St. Paul, Minn.

Rooks, S.D., J.R. Ballington, R.D. Milholland, W.O. Cline, and J.R. Meyer. 1995. Inventory of pest resistance in blueberry genotypes in North Carolina. J. Small Fruit Viticult. 3:99-110.

Searle, S.R., G. Casella, and C.E. McCulloch. 1992. Variance components. Wiley, New York

Smith, B.J. 2004. Susceptibility of southern highbush blueberry cultivars to botryosphaeria stem blight. Small Fruits Rev. 3:193-201.

Wilcox, M. 1939. Phomopsis twig blight of blueberry. Phytopathology 29:136-142.

Witcher, W. and C.N. Clayton. 1963. Blueberry stem blight caused by Botryosphaeria dothidea (B. Ribis). Phytopathology 53:705-712. 